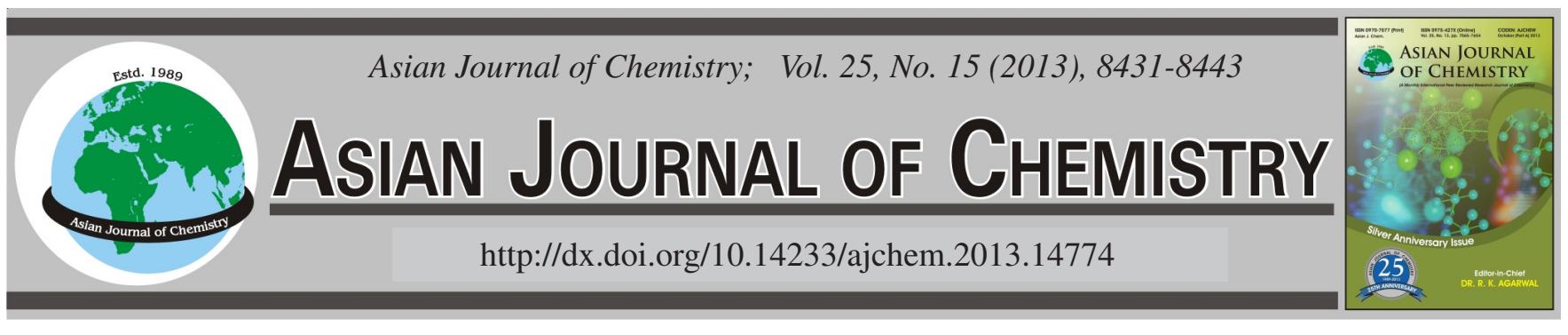

\title{
Effect of Acid-Base Property in Conjunction with Molecular Descriptors for Chemical Drugs and Bioactive Ingredients of Traditional Chinese Medicine on Prediction of Oral Absorption by Biopartioning Micellar Chromatography
}

\author{
Han $\mathrm{Chen}^{1}$, Yan Liang ${ }^{1}, \mathrm{Xiao} \mathrm{Juan} \mathrm{Ren}^{1}$, Dong Mei Xiong ${ }^{1}$, Shu Rong Wang ${ }^{2}$, Li Ping Wu ${ }^{3}$, Cong Chen ${ }^{1}$ and Li Ming Ye ${ }^{1, *}$
}

${ }^{1}$ West China School of Pharmacy, Sichuan University, Chengdu 610041, P.R. China

${ }^{2}$ Department of Pharmacy, The Affiliated Hospital of Luzhou Medical College, Luzhou 646000, P.R. China

${ }^{3}$ Chengdu Women and Children Central Hospital, Chengdu 610041, P.R. China

*Corresponding author: Fax: +86 28 85502305; Tel: +86 13980986791; E-mail: yeliminglaoshi@ 126.com

(Received: 3 November 2012;

Accepted: 23 August 2013)

AJC-13968

\begin{abstract}
This research was aimed to predict per cent of human oral absorption (HOA \%) for drugs by biopartitioning micellar chromatography (BMC). A data set of 102 chemical drugs containing acidic, basic, neutral and amphiprotic drugs from various structure classes with oral absorption rate data available was studied to construct prediction models of oral absorption for all chemical drugs, acidic chemical drugs, basic chemical drugs and neutral chemical drugs respectively and proved the effect of the acid-base property of drugs on oral absorption. Oral absorption was reciprocally correlated to the negative value of the capacity factor $(-1 / \mathrm{k})$. The correlation was improved with the addition of molecular descriptors. According to established prediction models for chemical drugs, biopartitioning micellar chromatography was applied to predict oral absorption of the organic acids and alkaloids of traditional Chinese medicines (TCM) for bioactive ingredients basing on constructed quantitative structure-activity relationship/quantitative retention-activity relationship models of chemical drugs. The results showed that application was predictable and practical in bioactive ingredients of traditional Chinese medicines.
\end{abstract}

Key Words: Biopartioning micellar chromatography, Oral absorption, Traditional Chinese medicines.

\section{INTRODUCTION}

Most commercially available drugs are administered orally as this noninvasive method is the preferred route of drug administration. Thus, understanding which properties need to be optimized in order to enhance oral absorption is quite basilic. The passive transcellular permeability of a compound that is crucial for its oral absorption is often modeled on lipophilicity, molecular size and/or hydrogen bonding ${ }^{1}$. Studies have unambiguously demonstrated that lipophilicity is a key parameter in predicting and interpreting permeability. Lipophilicity commonly expressed by the octanol/water partition coefficient as $\log \mathrm{P}$, has been successfully used to predict passive drug absorption in vivo for series of homologous compounds ${ }^{2}$. But it fails to encode some important recognition forces, such as ionic bonds, which are notably important because charged forms of some molecules are able to partition into phospholipids bilayer $^{3}$. For this reason, we must take the ionization of drugs under physiological environment into account according to their acid-base property. Lu et al. ${ }^{4}$ amended linear solvation energy relationship (LSER) by the introduction of a molecular electronic factor to establish quantitative structure-retention relationship of biopartitioning micellar chromatography (BMC) system. It was found that when the mean net charge per molecule (d) was introduced into LSER as the sixth variable, the LSER regression coefficients and predictive capability were significantly improved ${ }^{4}$. Moreover, the lipophilicity itself is inadequate to estimate solute's ability to penetrate a membrane barrier. Several studies have also shown that physico-chemical descriptors of molecules such as lipophilicity, polar surface area (PSA) and hydrogen bonding descriptors correlate with cell culture permeability and intestinal absorption ${ }^{5,6}$. Num_H_Donors_Lipinki (NHDL) is the number of hydrogen bond donors (a donor is any $\mathrm{O}-\mathrm{H}$ or $\mathrm{N}-\mathrm{H}$ group), yielding a poor permeability to biomembrane if large than 5 , according to Lipinski's 'rule of $5^{, 7}$. Molecular polar surface area has been shown to correlate very well with the human intestinal absorption, Caco- 2 monolayer permeability ${ }^{8,9}$. But the polar surface area has been demonstrated a non-linear relationship with permeability, which declines sigmoidally as polar surface area increases ${ }^{10}$.

Chromatographic models are universally used to study passive absorption due to experimental simplicity, low cost, accuracy and high throughput, among which the immobilized 
artificial membrane (IAM), immobilized-liposome chromatography (ILC) and biopartitioning micellar chromatography (BMC) system are well recognized models. Biopartitioning micellar chromatography is a chromatographic modality optimized in order to describe the biological behaviour of drugs which usually is comprised of a $\mathrm{C}_{18}$ reversed stationary phase and polyoxyethylene (23) lauryl ether (Brij35) micellar mobile phases in adequate experimental conditions provides a partitioning environment that can be used as a system that mimics drug biopartitioning. It has been testified to be useful to study human oral absorption prediction, chemical toxicity, skin permeability and penetration of drugs across the bloodbrain barrier $(\mathrm{BBB})^{11-16}$. Moreover, biological activity of different oral drugs were studied by our research group ${ }^{17-22}$.

Traditional Chinese medicines (TCM) makes great contributions for the prosperity and proliferation of people as their successful treatment and prevent for various diseases. They are usually very complex mixtures containing up to hundreds or even thousands of different ingredients and thus differ greatly from synthetic drugs in some aspects. So screening and analysis of bioactive ingredients of traditional Chinese medicines are very important not only for quality control of the crude drugs but also for elucidating the principles of therapeutic action of traditional Chinese medicines ${ }^{23}$. Usually, traditional methods for screening bioactive ingredients of traditional Chinese medicines are mostly animals' models. The whole-animal model can, apparently, reveal the efficiency, side effects and toxicity of medicines, but it is time-consuming, arduous and inappropriate for direct screening bioactive ingredients of traditional Chinese medicines. However, traditional Chinese medicines have similar retention behaviour on biopartitioning micellar chromatography column for their physico-chemical properties and absorption mechanisms are analogous with chemical drugs. Therefore, bioactive properties of traditional Chinese medicines can be predicted by biopartitioning micellar chromatography for its simplicity, low cost, accuracy and high throughput.

But when biopartitioning micellar chromatography is used to predict oral absorption of bioactive ingredients of traditional Chinese medicines, the retention may not be obtained accurately because of interference of other ineffective and unknown components. Therefore, in this paper, parts of monomers of traditional Chinese medicines for bioactive ingredients with available literatures about their oral absorption condition were just preliminarily predicted. Firstly, we constructed prediction models of chemical drugs for oral absorption, which were introduced to retention factor $(\mathrm{k})$ and molecular structure descriptors (describe molecular structure mathematically) of selected chemical drugs according to their acid-base property. And then, we predicted oral absorption using biopartitioning micellar chromatography basing on previously mature studies. Finally, prediction oral absorption of monomers of traditional Chinese medicines for bioactive ingredients by models of chemical drugs and comparison predicted value with literatures reported value of their oral absorption were conducted.

\section{EXPERIMENTAL}

The mobile phase consisted of $0.04 \mathrm{~mol} / \mathrm{L}$ polyoxyethylene (23) lauryl ether (Brij35, Acros, NJ, USA)) with $0.01 \mathrm{~mol} / \mathrm{L}$ sodium dihydrogenphosphate (analytical-reagent grade, Kelong, Chengdu, China) and was adjusted to $\mathrm{pH} 7.4$ which is the plasmatic $\mathrm{pH}$ value by sodium hydroxide. In order to reproduce the osmotic pressure of biological fluids, Sodium chloride (9.20 g/L, analytical-reagent grade, Kelong, Chengdu, China) was added to the micellar mobile phase. The sodium chloride concentration was close to physiological concentration of biological fluids. Water was from a millipore (Billerica, MA, USA) synergy ${ }^{\mathrm{TM}} 185$ system and was degassed before HPLC. The mobile phases injected into the chromatograph were filtered through $0.45 \mu \mathrm{m}$ micro porous membrane.

Among the 102 medicine drugs, piroxicam, isoniazid, verapamil, caffeine, meloxicam, hydrochlorothiazide, bumetanide, furosemide, pindolol were used as control articles provided by analysis test center of West China School of Pharmacy and the others were crude drug or formulations which would not affect their retention behaviour in this study because of the dilution of the mobile phase, donated by the pharmaceutical and pharmaceutical chemistry laboratories of West China School of Pharmacy, Sichuan University (Chengdu, China). Acidic reference substances of traditional Chinese medicines including chlorogenic acid, ferulic acid, caffeic acid, cinnamic acid, rhein, salvianolic acid $\mathrm{B}$, tanshinol and basic reference substances of traditional Chinese medicines including barbering, colchicinea, hypaconitine, theopylline, sinomenine, colchicines, tetrahydropalmatine which were purchased from National Institute for the control of Pharmaceutical and Biological Products.

Water-soluble drugs were dissolved in mobile phase solution. Lipophilic drugs were first dissolved in methanol (analytical-reagent grade, Kelong, Chengdu, China) and then were diluted with water to get appropriate concentration. Stock standard solutions of reference substance and crude drug of analytes were prepared by dissolving $10 \mathrm{mg}$ of the compound in $10 \mathrm{~mL}$ volumetric flask. Working solutions were prepared by dilution of the stock standard ones using the Brij35 solution. For those pharmaceutical preparations of analytes, working solutions were prepared by dissolving $10 \mathrm{mg}$ of the tablet or capsule powders of the drugs in $10 \mathrm{~mL}$ volumetric flask, then centrifuged at $1000 \times 10 \mathrm{rpm}$ for $5 \mathrm{~min}$. The working solutions injected into the chromatograph were filtered through $0.45 \mu \mathrm{m}$ microporous membranes (Xinya, Shanghai, China), respectively. All the solutions were stored under refrigeration at $4{ }^{\circ} \mathrm{C}$ before analysis.

The retention of drugs was measured using an LC-6A chromatograph with an LC-6A pump, an SPD-6AV UVvisible detector and a CTO-6A column thermostat (Shimadzu, Japan). Data were collected and processed on a Compaq computer installed with HP-Chemstation software (A0402, 1996). The solutions were injected into the chromatograph through a Rheodyne valve (Cotati, CA, USA), with a $20 \mu \mathrm{L}$ loop. The HPLC column was a Kromasil $\mathrm{C}_{18}$ column $(5 \mu \mathrm{m}$, $150 \times 4.6 \mathrm{~mm}$ i.d.) with a phenomenex security Guard ${ }^{\mathrm{TM}} \mathrm{C}_{18}$ guard cartridge. The mobile phase flow rate was $1.0 \mathrm{~mL} / \mathrm{min}$. The UV detection of chemical drugs was monitored at 220, 254, 270 and $300 \mathrm{~nm}$ and active ingredients of traditional Chinese medicines were set at 270, 280 and 240. 
All the assays were carried out at $37^{\circ} \mathrm{C}$ for simulating human body temperature. The retention data in biopartitioning micellar chromatography were calculated as capacity factors, $\mathrm{k}=\left(\mathrm{t}_{\mathrm{r}}-\mathrm{t}_{0}\right) / \mathrm{t}_{0}$, where $\mathrm{t}_{\mathrm{r}}$ is the retention time of the test compound and $t_{0}$ is the column dead time, which is the first fluctuation of baseline, determined by injecting water. The $\mathrm{k}$ values used in this study were the average value of triplicate. The retention data were highly reproducible.

Statistical analysis: The data set was analyzed using Microsoft ${ }^{\circledR}$ Excel 2003 (Microsoft Corporation). Multiple linear regression was used to carry out on the date set using SPSS 12.0 software (the SPSS for windows version 12.0, SPSS Inc. Chicago, USA). Stepwise regression analysis which is one of the MLR methods was used to determine the most significant descriptors. Molecular descriptors were calculated by Discovery Studio 2.5 (Accelrys Software Inc, San Diego, CA, USA). ChemDraw ${ }^{\circledR}$ Ultra 8.0.3 (Cambridge soft corporation, USA)was used. For each regression, the following requirements of significant regression analysis were observed: $n, R^{2}$, $\mathrm{SE}, \mathrm{F}$ and $\mathrm{p}$, in which $\mathrm{n}$ is the number of points used in the regression, $\mathrm{R}^{2}$ is the square of the overall correlation coefficient, $\mathrm{SE}$ is the standard deviation and F is Fischer's F-statistic, which are used to control fit ability and statistics significance of regression mode. T test is used to prove that partial regression coefficient before each variable is meaningful or not in multiple linear regression (MLR) equation. Variation inflation factor (VIF) determined the multicollinearities among the descriptors. Variation inflation factor was calculated for each descriptor in the model as $1 /\left(1-r^{2}\right)$. Multicollinearities were considered to exist when the variation inflation factor was greater than 10 and the model was considered to reconstruct. In addition, root mean squared error of calibration (RMSEC) was estimated predictive ability of the models.

\section{RESULTS AND DISCUSSION}

Retention/structure -oral absorption relationships of chemical drugs: For most drugs, the predominant mechanism of oral absorption is via the transcellular route. It relies on the ability of the molecule to partition into and move across gastrointestinal epithelium membranes. The most important features of a drug that influence this partitioning are solubility, permeability and molecular size, polarity and lipophilicity. A study suggests that $62.9 \%$ of drugs are ionizable, of which $14.5 \%$ are acids, $67.6 \%$ are bases and $17.9 \%$ are ampholytes of various types. Many drugs contain one or more ionizable groups and their lipophilicity is $\mathrm{pH}$ dependent. These biologically active molecules are at least partially ionized at biological $\mathrm{pH}$ values and absorption could be influenced by the membrane penetration of the neutral form and the receptor binding of the ionized form.

The first step in current study was to calculate bulk properties and molecular descriptors of the selected chemical drugs which were passively absorbed in intestinal wall, including neutral, acidic, basic and amphiprotic drugs with oral absorption $\%$ date available $e^{23-28}$ and to measure the retention (capacity factor k) of each drug on the biopartitioning micellar chromatography column as an indicator for the drug partitioning into cell membrane. The next step was to correlate calculated descriptors and experimentally measured capacity factor against oral absorption \%. Since an equation containing an excessive number of independent variables can be too cumbersome to use and is likely to be over parameterized, we utilized stepwise regression to refine the model and to select most important descriptors to generate regression equation of all chemical drugs, acidic chemical drugs, basic chemical drugs and natural chemical drugs, respectively. The findings of the study and their implications are elaborated in the subsequent sections.

All chemical drugs: The sample set of 102 chemical drugs with different acidity was random divided into training set including 84 drugs which was used to construct model and test set including 18 drugs which was used to evaluate predictability of model. The specific drugs, molecular descriptors, experimental oral absorption and predicted oral absorption calculated by equation (1) were showed in the Table-1 (supplementary files). The MLR equation was as follows:

Oral absorption $\%=114.163( \pm 4.63)-5.469( \pm 1.867) 1 / \mathrm{k}_{\mathrm{BMC}^{-}}$ $4.584( \pm 1.004)$ Num_H_Donors_Lipinki $-1.8151( \pm 0.0558)$ Kappa_2-8.727( \pm 2.757$)$ Num_Terminal Romoters

Ionizable drugs in the given data set are partly or fully in ionized form under experimental conditions. The retention behaviour of the neutral and ionized forms was quite different on the biopartitioning micellar chromatography column because an electrostatic force is involved in the interactions between the ionized form and the phospholipids membrane, but it was not the case with neutral compounds. As can be observed, the regression coefficient of equation $(1)\left(R^{2}=0.746\right)$ was a little poor and SE was slightly large, for which the following were listed as possible reasons: (1) Chemical drugs selected for constructing model of all chemical drugs are structurally divisive. The acidic chemical drugs and base chemical drugs may ionized partially at physiological $\mathrm{pH}$ and the retention behaviours on the biopartitioning micellar chromatography of neutral and ionized forms were quite different because electrostatic forces may be involved in the interaction between the ionized form and modified stationary phase of biopartitioning micellar chromatography, which might contribute to the poor relevance. (2) The oral absorption \% values -biopartitioning micellar chromatography model has some limitations that only relate the retention behaviour and molecular structure feature to the oral absorption \% values but it is physically unrealistic. It is unable to take the chemical and bacterial degradation of the drugs at the absorption site and the first-pass metabolism in the intestinal cells into consideration ${ }^{11}$. From the magnitude of coefficients indicted that Num_Terminal Romoters and 1/k were the two most important molecular descriptors determining oral absorption \% in the biopartitioning micellar chromatography system studied. The log k (at pH 7.0 or 7.4) is often considered as effective molecular descriptor, indicative of the potential absorption properties of drugs. The retention (capacity factor $\mathrm{k}$ ) on the biopartitioning micellar chromatography column is as an indicator for the drug partitioning into cell membrane. NTR are constitutional descriptors. Terminal rotomer is defined as either a non-terminal $s p^{3}$ atom connected to three-terminal atoms of the same type, or a non-terminal $s p^{2}$ atom connected to two-terminal atoms of the same type. The molecular descriptor $\mathrm{k}$ contributes the oral absorption $\%$ of the 
TABLE-1

MOLECULAR DESCRIPTORS, EXPERIMENTAL AND PREDICTED OA \% AND RESIDUAL VALUES (EXPERIMENTAL OA \% - PREDICTED ORAL ABSORPTION \%) OF ALL CHEMICAL DRUGS, ACIDIC CHEMICAL DRUGS, BASIC CHEMICAL DRUGS AND NATURAL CHEMICAL DRUGS

\begin{tabular}{|c|c|c|c|c|c|c|c|c|c|c|c|c|c|c|c|c|c|c|c|c|c|c|c|}
\hline & Compound & $1 / \mathrm{k}_{\mathrm{BMC}}$ & $\begin{array}{c}\mathrm{OA}_{\mathrm{Exp}} \\
(\%) \\
\end{array}$ & NHDL & $\begin{array}{c}\text { Kappa } \\
22 \\
\end{array}$ & NTR & $\begin{array}{c}\mathrm{OA}_{\text {Prel }} \\
(\%)\end{array}$ & $\mathrm{RV}_{1}$ & K3A & JY & NAR & NSB & NH & $\begin{array}{c}\mathrm{OA}_{\text {Pre2 }} \\
(\%)\end{array}$ & $\mathrm{RV}_{2}$ & IC & MS & NR5 & PSA & $\begin{array}{c}\mathrm{OA}_{\text {Pre3 }} \\
(\%)\end{array}$ & $\mathrm{RV}_{3}$ & $\begin{array}{c}\mathrm{OA}_{\text {Pre4 }} \\
(\%)\end{array}$ & $\mathrm{RV}_{4}$ \\
\hline \multicolumn{24}{|c|}{ Training set } \\
\hline 1 & Isoniazid & 1.72 & 20 & 7 & 13.98 & 0 & 29.84 & -9.84 & 1.53 & 2.89 & 1 & 0 & 0 & 16.14 & 3.86 & - & - & - & - & - & - & - & - \\
\hline 2 & Amitriptyline & 0.01 & 95 & 0 & 7.51 & 0 & 100.11 & -5.11 & 3.09 & 2.07 & 2 & 1 & 1 & 96.38 & -1.38 & - & - & - & - & - & - & - & - \\
\hline 3 & Ondansetron & 0.1 & 100 & 0 & 6.14 & 0 & 101.25 & -1.25 & 1.94 & 1.78 & 3 & 0 & 0 & 99.56 & 0.44 & - & - & - & - & - & - & - & - \\
\hline 4 & Aminophenazoe & 0.14 & 100 & 0 & 5.33 & 0 & 102.13 & -2.13 & 1.75 & 2.43 & 1 & 1 & 1 & 96.45 & 3.55 & - & - & - & - & - & - & - & - \\
\hline 5 & Oxazepam & 0.04 & 97 & 2 & 6.41 & 0 & 92.58 & 4.42 & 2.47 & 2.25 & 2 & 1 & 1 & 96.25 & 0.75 & - & - & - & - & - & - & - & - \\
\hline 6 & Aprobarbital & 0.09 & 85 & 2 & 4.89 & 0 & 94.62 & -9.62 & 2.95 & 3.18 & 0 & 1 & 1 & 91.82 & -6.82 & - & - & - & - & - & - & - & - \\
\hline 7 & Phenylbutazone & 0.1 & 90 & 0 & 8.39 & 0 & 97.09 & -7.09 & 2.84 & 2.04 & 2 & 0 & 0 & 85 & 5.00 & - & - & - & - & - & - & - & - \\
\hline 8 & Benazepril & 0.12 & 37 & 5 & 12.89 & 2 & 48.25 & -11.25 & 6.23 & 1.45 & 2 & 0 & 0 & 38.47 & -1.47 & - & - & - & - & - & - & - & - \\
\hline 9 & Phenobarbital & 0.08 & 100 & 2 & 5.33 & 0 & 93.96 & 6.04 & 1.6 & 2.56 & 1 & 0 & 0 & 94.55 & 5.45 & - & - & - & - & - & - & - & - \\
\hline 10 & Phenytoin & 0.05 & 98 & 2 & 5.78 & 0 & 93.59 & 4.41 & 1.69 & 2.25 & 2 & 0 & 0 & 99.22 & -1.22 & - & - & - & - & - & - & - & - \\
\hline 11 & Piroxicam & 0.12 & 99 & 2 & 7.09 & 0 & 90.09 & 8.91 & 2.47 & 2.11 & 2 & 1 & 1 & 89.85 & 9.15 & - & - & - & - & - & - & - & - \\
\hline 12 & Probenecid & 0.13 & 90 & 1 & 7.7 & 1 & 84.62 & 5.38 & 3.86 & 3.08 & 1 & 0 & 0 & 88.41 & 1.59 & - & - & - & - & - & - & - & - \\
\hline 13 & Ibuprofen & 0.08 & 95 & 1 & 5.92 & 1 & 88.69 & 6.31 & 3.48 & 2.77 & 1 & 0 & 0 & 85.29 & 9.71 & - & - & - & - & - & - & - & - \\
\hline 14 & Diazepam & 0.03 & 100 & 0 & 6.41 & 0 & 101.83 & -1.83 & 2.36 & 2.24 & 2 & 1 & 1 & 99.46 & 0.54 & - & - & - & - & - & - & - & - \\
\hline 15 & Felodipine & 0.06 & 100 & 1 & 9.27 & 0 & 91.53 & 8.47 & 3.8 & 2.76 & 1 & 2 & 2 & 95.35 & 4.65 & - & - & - & - & - & - & - & - \\
\hline 16 & Flurbiprofen & 0.19 & 95 & 1 & 6.44 & 1 & 86.03 & 8.97 & 2.55 & 2.44 & 2 & 0 & 0 & 89.02 & 5.98 & - & - & - & - & - & - & - & - \\
\hline 17 & Flumazenil & 0.11 & 95 & 0 & 6.86 & 0 & 99.76 & -4.76 & 2.33 & 2.23 & 2 & 0 & 0 & 90.29 & 4.71 & - & - & - & - & - & - & - & - \\
\hline 18 & Furosemide & 0.14 & 67 & 4 & 7.05 & 1 & 72.03 & -5.03 & 3.61 & 2.22 & 2 & 0 & 0 & 71.74 & -4.74 & - & - & - & - & - & - & - & - \\
\hline 19 & Flunitrazepam & 0.04 & 100 & 0 & 7.49 & 1 & 90.95 & 9.05 & 2.62 & 2.3 & 2 & 1 & 1 & 96.94 & 3.06 & - & - & - & - & - & - & - & - \\
\hline 20 & Hexobarbital & 0.05 & 95 & 1 & 4.94 & 0 & 99.70 & -4.7 & 1.7 & 2.48 & 0 & 2 & 2 & 95.07 & -0.07 & - & - & - & - & - & - & - & - \\
\hline 21 & Warfarin sodium & 0.11 & 93 & 1 & 7.92 & 0 & 93.25 & -0.25 & 2.99 & 2.17 & 2 & 1 & 1 & 89.04 & 3.96 & - & - & - & - & - & - & - & - \\
\hline 22 & Ciprofloxacin & 0.11 & 69 & 2 & 6.96 & 1 & 81.76 & -12.76 & 2.73 & 1.82 & 1 & 1 & 1 & 70.43 & -1.43 & - & - & - & - & - & - & - & - \\
\hline 23 & Methylprednisolone & 0.06 & 82 & 3 & 6.5 & 0 & 87.56 & -5.56 & 2.28 & 1.79 & 0 & 2 & 2 & 80.57 & 1.43 & - & - & - & - & - & - & - & - \\
\hline 24 & Trimethoprim & 0.17 & 97 & 4 & 8.02 & 0 & 78.50 & 18.5 & 3.07 & 2.53 & 2 & 0 & 0 & 91.3 & 5.70 & - & - & - & - & - & - & - & - \\
\hline 25 & Lamotrigine & 0.08 & 98 & 4 & 5.1 & 0 & 85.29 & 12.71 & 2.03 & 2.65 & 2 & 0 & 0 & 101.11 & -3.11 & - & - & - & - & - & - & - & - \\
\hline 26 & Lorazepam & 0.05 & 93 & 2 & 6.63 & 0 & 92.02 & 0.98 & 2.65 & 2.28 & 2 & 1 & 1 & 93.75 & -0.75 & - & - & - & - & - & - & - & - \\
\hline 27 & Clobazam & 0.04 & 87 & 0 & 6.63 & 0 & 101.27 & -14.27 & 2.38 & 2.26 & 2 & 0 & 0 & 93.32 & -6.32 & - & - & - & - & - & - & - & - \\
\hline 28 & Chlordiazepoxide & 0.03 & 100 & 1 & 6.84 & 0 & 96.49 & 3.51 & 2.61 & 2.2 & 2 & 2 & 2 & 102.41 & -2.41 & - & - & - & - & - & - & - & - \\
\hline 29 & Lormetazepam & 0.04 & 98 & 1 & 6.86 & 0 & 96.30 & 1.700 & 2.64 & 2.32 & 2 & 1 & 1 & 97.3 & 0.7 & - & - & - & - & - & - & - & - \\
\hline 30 & Losartan & 0.1 & 80 & 2 & 11.23 & 0 & 82.73 & -2.73 & 4.3 & 1.63 & 4 & 0 & 0 & 85.92 & -5.92 & - & - & - & - & - & - & - & - \\
\hline 31 & Clonazepam & 0.05 & 98 & 1 & 7.27 & 1 & 86.66 & 11.34 & 2.77 & 2.25 & 2 & 1 & 1 & 91.56 & 6.44 & - & - & - & - & - & - & - & - \\
\hline 32 & Meloxicam & 0.09 & 90 & 2 & 6.72 & 0 & 91.24 & -1.24 & 2.52 & 2.1 & 2 & 1 & 1 & 90.56 & -0.56 & - & - & - & - & - & - & - & - \\
\hline 33 & Midazolam & 0.02 & 100 & 0 & 6.72 & 0 & 101.41 & -1.41 & 2.56 & 2.04 & 3 & 1 & 1 & 105.07 & -5.07 & - & - & - & - & - & - & - & - \\
\hline
\end{tabular}




\begin{tabular}{|c|c|c|c|c|c|c|c|c|c|c|c|c|c|c|c|c|c|c|c|c|c|c|c|}
\hline 34 & Minoxidil & 0.92 & 28 & 4 & 12.68 & 2 & 40.81 & -12.81 & 2.26 & 2.04 & 1 & 0 & 0 & 31.69 & -3.69 & - & - & - & - & - & - & - & - \\
\hline 35 & Naproxen & 0.14 & 99 & 1 & 5.76 & 1 & 88.06 & 10.94 & 2.35 & 2.5 & 2 & 0 & 0 & 96.35 & 2.65 & - & - & - & - & - & - & - & - \\
\hline 36 & Nitrendipine & 0.07 & 88 & 1 & 9.97 & 1 & 81.35 & 6.65 & 3.96 & 2.74 & 1 & 2 & 2 & 93.38 & -5.38 & - & - & - & - & - & - & - & - \\
\hline 37 & Pravastatin & 0.33 & 34 & 4 & 11.92 & 3 & 42.62 & -8.62 & 7.33 & 2.17 & 0 & 2 & 2 & 29.29 & 4.71 & - & - & - & - & - & - & - & - \\
\hline 38 & Amrinone & 0.24 & 93 & 3 & 4.68 & 0 & 88.14 & 4.86 & - & - & - & - & - & - & - & 3.33 & -1.94 & 0 & 68 & 90.52 & 2.48 & - & - \\
\hline 39 & AmLodipine & 0.05 & 80 & 3 & 12 & 0 & 77.53 & 2.47 & - & - & - & - & - & - & - & 4 & -4.6 & 0 & 100 & 80.93 & -0.9 & - & - \\
\hline 40 & Olanzapine & 0.21 & 91 & 1 & 6.48 & 0 & 94.37 & -3.37 & - & - & - & - & - & - & - & 3.73 & -3.67 & 1 & 59 & 89.88 & 1.12 & - & - \\
\hline 41 & Omeprazole & 0.05 & 80 & 1 & 8.13 & 0 & 93.79 & -13.79 & - & - & - & - & - & - & - & 3.96 & -4.91 & 1 & 98 & 85.22 & -5.2 & - & - \\
\hline 42 & Alprenolol & 0.05 & 93 & 2 & 8.99 & 0 & 87.65 & 5.35 & - & - & - & - & - & - & - & 3.31 & -3.72 & 0 & 41 & 93.48 & -0.5 & - & - \\
\hline 43 & Atenolol & 1.96 & 56 & 4 & 9.03 & 0 & 48.94 & 7.06 & - & - & - & - & - & - & - & 3.22 & -2.86 & 0 & 85 & 58.67 & -2.7 & - & - \\
\hline 44 & Allopurinol & 0.85 & 90 & 2 & 2.56 & 0 & 87.18 & 2.82 & - & - & - & - & - & - & - & 2.65 & -0.17 & 1 & 75 & 92.4 & -2.4 & - & - \\
\hline 45 & Prilocaine & 0.12 & 90 & 2 & 7.35 & 0 & 89.61 & 0.39 & - & - & - & - & - & - & - & 3.75 & -3.46 & 0 & 41 & 88.88 & 1.12 & - & - \\
\hline 46 & Imipramine & 0.02 & 95 & 0 & 7.51 & 0 & 99.95 & -4.95 & - & - & - & - & - & - & - & 3.15 & -4.52 & 0 & 6.5 & 97.19 & -2.2 & - & - \\
\hline 47 & Bupivacaine & 0.03 & 80 & 1 & 8.59 & 0 & 93.25 & -13.25 & - & - & - & - & - & - & - & 3.99 & -5.71 & 0 & 39 & 84.91 & -4.9 & - & - \\
\hline 48 & Scopolamine & 0.38 & 95 & 1 & 6.14 & 0 & 92.37 & 2.63 & - & - & - & - & - & - & - & 3.57 & -1.45 & 1 & 62 & 91.6 & 3.4 & - & - \\
\hline 49 & Irbesartan & 0.06 & 92 & 1 & 10.73 & 0 & 88.83 & 3.17 & - & - & - & - & - & - & - & 3.58 & -6.84 & 3 & 87 & 94.85 & -2.9 & - & - \\
\hline 50 & Irbesartan & 2.78 & 34 & 5 & 3.92 & 2 & 23.71 & 10.29 & - & - & - & - & - & - & - & 2.73 & 1.59 & 0 & 131 & 30.94 & 3.06 & - & - \\
\hline 51 & Fluphenazine & 1.79 & 65 & 1 & 9.67 & 0 & 64.03 & 0.97 & - & - & - & - & - & - & - & 3.43 & -4.13 & 0 & 55 & 60.35 & 4.65 & - & - \\
\hline 52 & Haloperidol & 0.02 & 95 & 1 & 9.47 & 0 & 91.78 & 3.22 & - & - & - & - & - & - & - & 3.08 & -5.7 & 0 & 41 & 92.91 & 2.09 & - & - \\
\hline 53 & Labetalol & 0.07 & 90 & 5 & 10.22 & 0 & 71.42 & 18.58 & - & - & - & - & - & - & - & 3.19 & -4.74 & 0 & 96 & 87.78 & 2.22 & - & - \\
\hline 54 & Ranitidine & 1.41 & 50 & 2 & 10.68 & 1 & 54.76 & -4.76 & - & - & - & - & - & - & - & 3.79 & -3.9 & 1 & 128 & 51.7 & -1.7 & - & - \\
\hline 55 & Ribavirin & 3.57 & 33 & 5 & 5.33 & 0 & 26.33 & 6.67 & - & - & - & - & - & - & - & 3.74 & -0.25 & 2 & 144 & 33.78 & -0.8 & - & - \\
\hline 56 & Metoprolol & 0.33 & 90 & 2 & 9.83 & 0 & 81.77 & 8.23 & - & - & - & - & - & - & - & 3.12 & -3.43 & 0 & 51 & 89.92 & 0.08 & - & - \\
\hline 57 & Nadolol & 1.69 & 40 & 4 & 7.71 & 1 & 46.83 & -6.83 & - & - & - & - & - & - & - & 3.98 & -3.19 & 0 & 129 & 41.9 & -1.9 & - & - \\
\hline 58 & Norfloxacin & 0.11 & 71 & 2 & 7.49 & 1 & 80.77 & -9.77 & - & - & - & - & - & - & - & 3.98 & -2.44 & 0 & 76 & 75.22 & -4.2 & - & - \\
\hline 59 & Procaine & 0.26 & 85 & 3 & 8.16 & 0 & 81.39 & 3.61 & - & - & - & - & - & - & - & 3.38 & -2.43 & 0 & 58 & 89.81 & -4.8 & - & - \\
\hline 60 & Propranolol & 0.23 & 90 & 2 & 7.7 & 0 & 87.26 & 2.74 & - & - & - & - & - & - & - & 3.25 & -4.16 & 0 & 41 & 90.17 & -0.2 & - & - \\
\hline 61 & Zidovudine & 0.45 & 100 & 2 & 6.64 & 0 & 85.82 & 14.18 & - & - & - & - & - & - & - & 3.38 & -0.3 & 1 & 20 & 97.6 & 2.4 & - & - \\
\hline 62 & Nortriptyline & 0.03 & 95 & 1 & 7.32 & 0 & 95.6 & -0.6 & - & - & - & - & - & - & - & 3.22 & -6.27 & 0 & 12 & 93.11 & 1.89 & - & - \\
\hline 63 & Tramadol & 0.14 & 90 & 1 & 6.64 & 0 & 95.16 & -5.16 & - & - & - & - & - & - & - & 3.93 & -3.34 & 0 & 33 & 87.91 & 2.09 & - & - \\
\hline 64 & Timolol & 0.33 & 90 & 2 & 8.02 & 1 & 76.39 & 13.61 & - & - & - & - & - & - & - & 3.17 & -0.7 & 1 & 101 & 83.32 & 6.68 & - & - \\
\hline 65 & Sulpiride & 0.67 & 86 & 3 & 8.39 & 0 & 74.62 & 11.38 & - & - & - & - & - & - & - & 3.27 & -2.69 & 1 & 110 & 82.98 & 3.02 & - & - \\
\hline 66 & Terbutaline & 0.65 & 73 & 4 & 5.56 & 1 & 66.90 & 6.1 & - & - & - & - & - & - & - & 2.85 & -2.1 & 0 & 73 & 76.49 & -3.5 & - & - \\
\hline 67 & Verapami & 0.02 & 90 & 0 & 14.53 & 0 & 86.96 & 3.04 & - & - & - & - & - & - & - & 3.35 & -7.51 & 0 & 64 & 85.6 & 4.4 & - & - \\
\hline 68 & Pentobarbital & 0.02 & 100 & 2 & 5.56 & 0 & 94.47 & 5.53 & - & - & - & - & - & - & - & 3.33 & -2.53 & 0 & 35 & 96.28 & 3.72 & - & - \\
\hline 69 & Urapidil & 0.2 & 78 & 1 & 10.86 & 0 & 86.42 & -8.42 & - & - & - & - & - & - & - & 3.99 & -4.1 & 0 & 68 & 81.99 & -4 & - & - \\
\hline 70 & Cimetidine & 0.33 & 84 & 3 & 9 & 0 & 78.76 & 5.24 & - & - & - & - & - & - & - & 3.57 & -2.92 & 1 & 114 & 85.54 & -1.5 & - & - \\
\hline 71 & Bromperidol & 0.02 & 95 & 1 & 9.47 & 0 & 91.78 & 3.22 & - & - & - & - & - & - & - & 3.08 & -6.12 & 0 & 41 & 92.24 & 2.76 & - & - \\
\hline
\end{tabular}




\begin{tabular}{|c|c|c|c|c|c|c|c|c|c|c|c|c|c|c|c|c|c|c|c|c|c|c|c|}
\hline 72 & Pindolol & 0.29 & 87 & 3 & 6.96 & 0 & 83.15 & 3.85 & - & - & - & - & - & - & - & 3.57 & -3.01 & 1 & 57 & 91.1 & -4.1 & - & - \\
\hline 73 & Amobarbital & 0.03 & 95 & 2 & 5.56 & 0 & 94.31 & 0.69 & - & - & - & - & - & - & - & 3.33 & -2.44 & 0 & 45 & 95.37 & -0.4 & - & - \\
\hline 74 & Aciclovir & 2.56 & 30 & 4 & 5.56 & 0 & 46.08 & -16.08 & - & - & - & - & - & - & - & 4.55 & - & - & - & - & - & - & - \\
\hline 75 & Theophylline & 0.63 & 89 & 1 & 3.29 & 0 & 93.78 & -4.78 & - & - & - & - & - & - & - & 2.085 & - & - & - & - & - & - & - \\
\hline 76 & Digoxin & 0.43 & 81 & 1 & 8.79 & 1 & 77.97 & 3.03 & - & - & - & - & - & - & - & - & - & - & - & - & 84.14 & - & - \\
\hline 77 & Dexamethasone & 0.04 & 92 & 3 & 6.5 & 0 & 87.87 & 4.13 & - & - & - & - & - & - & - & 3.893 & - & - & - & - & - & - & - \\
\hline 78 & Mannitol & 4.76 & 16 & 3 & 5.61 & 0 & 16.50 & -0.5 & - & - & - & - & - & - & - & 1.892 & - & - & - & - & - & - & - \\
\hline 79 & Testosterone & 0.02 & 100 & 1 & 4.75 & 0 & 100.51 & -0.51 & - & - & - & - & - & - & - & 3.74 & - & - & - & - & - & - & - \\
\hline 80 & Caffeine & 0.47 & 92 & 0 & 3.54 & 0 & 100.34 & -8.34 & - & - & - & - & - & - & - & 2.836 & - & - & - & - & - & - & - \\
\hline 81 & Prazosin & 0.07 & 100 & 2 & 9.43 & 0 & 86.53 & 13.47 & - & - & - & - & - & - & - & 3.023 & - & - & - & - & - & - & - \\
\hline 82 & Corticosterone & 0.03 & 100 & 2 & 6.27 & 0 & 93.00 & 7 & - & - & - & - & - & - & - & 3.569 & - & - & - & - & - & - & - \\
\hline 83 & Primidone & 0.14 & 90 & 2 & 5.10 & 0 & 93.46 & -3.46 & - & - & - & - & - & - & - & 3.603 & - & - & - & - & - & - & - \\
\hline 84 & Hydrocortisone & 0.21 & 89 & 3 & 6.25 & 0 & 85.70 & 3.3 & - & - & - & - & - & - & - & 3.779 & - & - & - & - & - & - & - \\
\hline 85 & Quinine & 0.07 & 93 & 1 & 7.32 & 0 & 94.98 & -1.98 & - & - & - & - & - & - & - & 4.085 & - & - & - & - & - & - & - \\
\hline \multicolumn{24}{|c|}{ Test set } \\
\hline 1 & Alprazolam & 0.04 & 95 & 0 & 8.481 & 0 & 97.86 & -2.86 & 2.96 & 2.03 & 3 & 1 & 1 & 99.3 & -4.3 & - & - & - & - & - & - & - & - \\
\hline 2 & Phenazone & 0.31 & 97 & 0 & 4.245 & 0 & 101.56 & -4.56 & 1.4 & 2.57 & 1 & 1 & 1 & 91.34 & 5.66 & - & - & - & - & - & - & - & - \\
\hline 3 & Bumetanide & 0.1 & 100 & 1 & 3.196 & 1 & 93.36 & 6.64 & 5.1 & 3.51 & 2 & 0 & 0 & 102.53 & -2.53 & - & - & - & - & - & - & - & - \\
\hline 4 & Propylthiouracil ethyl & 0.3 & 76 & 1 & 12.133 & 0 & 82.45 & -6.45 & 2.01 & 2.63 & 0 & 1 & 1 & 71.74 & 4.26 & - & - & - & - & - & - & - & - \\
\hline 5 & Fluvastatin & 0.08 & 100 & 1 & 3.228 & 1 & 93.6 & 6.4 & 5.04 & 2.39 & 3 & 1 & 1 & 96.14 & 3.86 & - & - & - & - & - & - & - & - \\
\hline 6 & Carbamazepine & 0.07 & 100 & 2 & 5.551 & 0 & 93.64 & 6.36 & 1.7 & 2.12 & 2 & 1 & 1 & 100.08 & -0.08 & - & - & - & - & - & - & - & - \\
\hline 7 & Hydrochlorothiazide & 0.1 & 90 & 4 & 4.281 & 0 & 86.5 & 3.5 & 2.24 & 2.79 & 1 & 0 & 0 & 89.76 & 0.24 & - & - & - & - & - & - & - & - \\
\hline 8 & Valsartan & 0.28 & 55 & 5 & 13.185 & 1 & 53.88 & 1.12 & 5.66 & 1.79 & 3 & 0 & 0 & 58.24 & -3.24 & - & - & - & - & - & - & - & - \\
\hline 9 & Lamivudine & 1.28 & 87 & 2 & 2.888 & 0 & 79.89 & 7.11 & 1.04 & 3.64 & 0 & 2 & 2 & 80.68 & 6.32 & - & - & - & - & - & - & - & - \\
\hline 10 & Amoxapine & 0.03 & 95 & 1 & 6.857 & 0 & 96.46 & -1.46 & - & - & - & - & - & - & - & 3.61 & -4.15 & 0 & 37 & 90.97 & 4.03 & - & - \\
\hline 11 & Orphenadrine & 0.02 & 95 & 0 & 8.444 & 0 & 98.22 & -3.22 & - & - & - & - & - & - & - & 3.54 & -4.78 & 0 & 12 & 92.85 & 2.15 & - & - \\
\hline 12 & Atropine & 0.24 & 98 & 1 & 7.051 & 0 & 92.92 & 5.08 & - & - & - & - & - & - & - & 3.59 & -2.47 & 1 & 50 & 93.44 & 4.56 & - & - \\
\hline 13 & Paracetamol & 0.26 & 80 & 3 & 5.133 & 0 & 86.98 & -6.98 & - & - & - & - & - & - & - & 3.99 & -3.98 & 0 & 59 & 81.91 & -1.9 & - & - \\
\hline 14 & Lidocaine & 0.08 & 90 & 1 & 7.438 & 0 & 94.56 & -4.56 & - & - & - & - & - & - & - & 3.70 & -3.2 & 0 & 32 & 91.16 & -1.2 & - & - \\
\hline 15 & Venlafaxine & 0.23 & 97 & 1 & 7.320 & 0 & 92.43 & 4.57 & - & - & - & - & - & - & - & 3.00 & -3.88 & 0 & 33 & 93.49 & 3.51 & - & - \\
\hline 16 & Oxprenolol & 0.13 & 97 & 2 & 6.834 & 0 & 90.35 & 6.65 & - & - & - & - & - & - & - & 3.06 & -3.23 & 0 & 51 & 94.21 & 2.79 & - & - \\
\hline 17 & Chlorpromazine & 0.01 & 95 & 0 & 7.951 & 0 & 99.25 & -4.25 & - & - & - & - & - & - & - & 3.34 & -4.85 & 0 & 32 & 92.96 & 2.04 & - & - \\
\hline \multicolumn{24}{|c|}{ 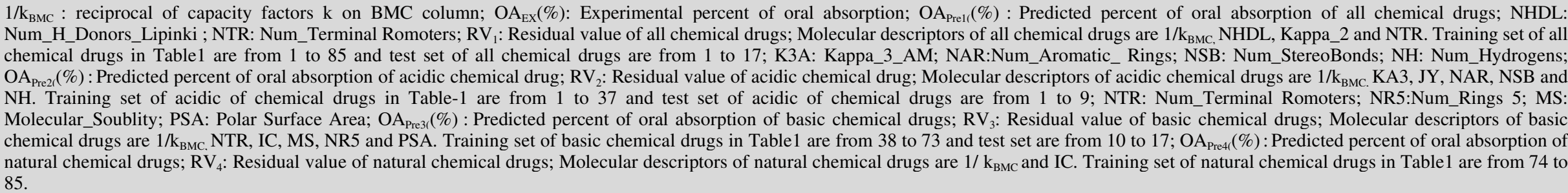 } \\
\hline
\end{tabular}


compounds and the molecular descriptor NTR will reduce the oral absorption \%.

Acidic chemical drugs: A sample set including 46 diverse structural acidic chemical drugs was random divided into training set with 37 acidic chemical drugs and test set with 9 . The specific drugs, molecular descriptors, experimental oral absorption \% and predicted oral absorption \% calculated by equation (2) were shown in the Table-1 (supplementary files). The MLR equation was as follows:

Oral absorption \% = $18.362( \pm 19.763)-1.416( \pm 4.500) 1 /$ $\mathrm{k}_{\mathrm{BMC}}-10.986( \pm 1.743)$ KAPPA_3_AM $+28.975( \pm 5.640) J \mathrm{Y}$ $+13.095( \pm 2.614)$ Num_Aromatic_Rings5 + $5.977( \pm 2.062)$ Num_StereoBonds $+0.884( \pm 0.412)$ Num_Hydrogens $\mathrm{N}=37 ; \mathrm{R}^{2}=0.906 ; \mathrm{SE}=7.26 ; \mathrm{F}=48.13 ; \mathrm{P}<0.001$

Acidic chemical drugs mostly contain carboxylic group $(-\mathrm{COOH})$, the other does not have such a group. Lu et al. ${ }^{4}$ found that the dissociation of the carboxylic group had a great impact on the retention of the acidic drugs with such a group on biopartitioning micellar chromatography. Its dissociation decreased the retention of acidic drugs because of strong polarity and weak fat-soluble of molecule in the dissociation state leading to difficulty transmembrane diffusion ${ }^{4}$. Besides, such categories of clonazepam and lorazepam, they are without carboxylic group in their structures indeed had long experimental retention. This seemed to show strong interactions between this ionizable molecule and the biopartitioning micellar chromatography column. Experimental errors and spatial matching might contribute to this strong retention. The regression coefficient increased from 0.746 to 0.928 , suggesting the consideration of acid-base property is necessary. In addition, the coefficient of KAPPA_3_AM (K3A) and JY are larger than other molecular descriptors in magnitudes suggesting that K3A and JY impacting most on the oral absorption $\%$ of acid drugs. K3A is $\alpha$ modified shape index of order three. The $\alpha$-modified Kappa shape indices are refinements of the kappa shape indices that take into the contribution of covalent radii and hybridization states consideration, reflecting the shape characteristic of molecule as well. JY is one of Balaban Indices belonging to topological descriptors, describes connective and branch information of atoms composed of in molecules and impacts the steric and hydrophobic function of molecule. The negative coefficient of K3A suggests that molecular shape can not allowed good absorption. And JY is positive to absorption.

Basic chemical drugs: A sample set including 44 diverse structural basic chemical drugs is random divided into training set with 36 basic chemical drugs and test set with 8 . The specific drugs, molecular descriptors, experimental oral absorption and predicted oral absorption obtained from flowing equation (3) with 6 parameters by means of MLR method were showed in Table-1 (supplementary files).

Oral absorption $\%=132.737( \pm 9.808)-17.368( \pm 1.554) 1 /$ $\mathrm{k}_{\mathrm{BMC}}-10.420( \pm 2.611)$ Num_Terminal Romoters $-8.694( \pm$ $2.987) \mathrm{IC}+1.603( \pm 0.562)$ Molecular_Soublity $+4.305( \pm$ 1.513)Num_Rings5-0.088 ( \pm 0.042) Polar Surface Area $\mathrm{N}=36 ; \mathrm{R}^{2}=0.929 ; \mathrm{SE}=5.27 ; \mathrm{F}=63.10 ; \mathrm{P}<0.001$

The pKa values of the basic drugs considered the range of 7-10.2. An ionizable compound can be assumed to exist in its total ionized form when $\mathrm{pH}$ value is and less than one unit away from $\mathrm{pKa}$ ( $\mathrm{pKa}-1$ for base).The retention of basic chemical drugs does not seem to be significantly affected by ionization; attractive interactions play an important role in the retention. Kotecha et al. ${ }^{1}$ performed an immobilized artificial membrane chromatographic method on the retention behaviours of 28 drugs represented acidic, basic, neutral and amphoteric drugs for predicting human oral absorption. The authors observed that basic drugs $(\mathrm{pKa}>8)$ that remain almost ionized ( 80 to $100 \%$ ) at $\mathrm{pH} 7.4$ showed lipophilicity based retention regardless of the degree of ionization. In another paper, Barbato et al. ${ }^{27}$ also concluded experimentally that even in the ionized form, the retention of basic drugs was as strong as if they were uncharged. From the magnitudes of coefficients, descriptors of $1 / \mathrm{k}_{\mathrm{BMC}}$ and NTR also impact mostly on oral absorption.

Neutral chemical drugs: This category consisted of 12 neutral chemical drugs with diverse structures and physicochemical characteristics. Inclusion criteria were used in the selection of the model drugs. These included the following: (1) neutral drugs without ionization at physiological condition; and (2) these drugs should be of oral delivery and the absorbed fractions in humans were available. So this model was developed using a relatively small training set. The value of oral absorption $\%$ of 12 kinds of neutral chemical drugs and corresponding molecular structure descriptors were seen in Table-1 (supplementary files).

Multiple linear regression was applied to construct optimal oral absorption \% prediction model of neutral chemical compound containing two independent variables.

$$
\text { Oral absorption } \%=138.347( \pm 18.963)-21.680
$$$$
( \pm 1.914) 1 / \mathrm{k}_{\mathrm{BMC}}-11.290( \pm 4.983) \mathrm{IC}
$$

$\mathrm{N}=12 ; \mathrm{R}^{2}=0.955 ; \mathrm{SE}=6.54 ; \mathrm{F}=94.778 ; \mathrm{P}<0.001$ (4)

Since Brij35 is non-ionic surfactant, natural chemical drugs can be allowed more retained than basic and acidic chemical drugs. As a result they can obtain good oral absorption consequence of easy transcellar diffusion.

As can be observed, the $p$-values obtained for four models (eqns. 1-4) indicated that the relationships between these parameters and the oral absorption \% were statistically significant at the $95 \%$ confidence level. The coefficients obtained for those models were also significant at the same confidence. When MLR was used to construct model each variable should perform $t$ test. From the Table-2 (supplementary files) listed, $t$ value of each variable was large than the standard value of $\mathrm{t}$ for four models (all chemical drugs, $\mathrm{t} \alpha / 2=1.99$; acidic chemical drugs, $t \alpha / 2=2.042$; basic chemical drugs, $t \alpha / 2=$ 2.045; natural chemical drugs, $t \alpha / 2=2.262$ ) at $95 \%$ confidence level, which indicated that each variable affected significantly to the oral absorption \%. Meanwhile, various inflation factor (VIF) calculated for each variable in the four models was less than 5, suggesting that the model was robust because multicollinearities among variables were not existing. Figs. 1c-3c and Fig. 4b (supplementary files) show the corresponding residual plots (Experimental oral absorption \% - Predicted oral absorption \%). There was a random distribution of the residuals and practically they were all statistically small which suggest, from a qualitative point of view, the adequacy of the 


\begin{tabular}{|c|c|c|c|}
\hline \multicolumn{4}{|c|}{$\begin{array}{l}\text { TABLE- } 2 \\
\text { VALUE OF t AND VARIATION INFLATION FACTOR } \\
\text { (VIF) OF EACH VARIABLE FOR EQNS. } 1-4\end{array}$} \\
\hline & Variables in each equation & VIF & $\mathrm{t}$ \\
\hline \multirow{4}{*}{$\begin{array}{l}\text { All } \\
\text { chemical } \\
\text { compounds }\end{array}$} & $1 / \mathrm{k}_{\mathrm{BMC}}$ & 1.615 & -8.285 \\
\hline & Num_H_Donors_Lipinki & 1.572 & -4.530 \\
\hline & Kappa_2 & 1.051 & -3.318 \\
\hline & Num_Terminal Romoters & 1.030 & -0.322 \\
\hline \multirow{6}{*}{$\begin{array}{l}\text { Acidic } \\
\text { chemical } \\
\text { compounds }\end{array}$} & $1 / \mathrm{k}_{\mathrm{BMC}}$ & 1.269 & -11.426 \\
\hline & KAPPA_3_AM & 2.931 & -6.308 \\
\hline & JY & 2.761 & 5.138 \\
\hline & Num_Aromatic_Rings & 3.426 & 5.010 \\
\hline & Num_Stereo Bonds & 1.592 & 2.898 \\
\hline & Num_Hydrogens Hydrogens & 4.717 & 2.145 \\
\hline \multirow{6}{*}{$\begin{array}{l}\text { Basic } \\
\text { chemical } \\
\text { compounds }\end{array}$} & $1 / \mathrm{k}_{\mathrm{BMC}}$ & 2.130 & -11.174 \\
\hline & Num_Terminal Romoters & 1.873 & -3.990 \\
\hline & IC & 1.367 & -2.911 \\
\hline & $\begin{array}{l}\text { Molecular_Solubility } \\
\text {-Solubility }\end{array}$ & 1.477 & 2.850 \\
\hline & Num_Rings 5 & 1.379 & 2.846 \\
\hline & Polar surface area & 2.779 & -2.090 \\
\hline \multirow{2}{*}{$\begin{array}{l}\text { Natural } \\
\text { chemical } \\
\text { compounds }\end{array}$} & $1 / \mathrm{k}_{\mathrm{BMC}}$ & 1.940 & -11.328 \\
\hline & IC & 1.940 & -2.266 \\
\hline
\end{tabular}

model to data. But some factors must be considered, such as appropriate molecular descriptors for each regression model and steric effect.

The predictive ability of the four models was evaluated in terms of RMSEC. Applying four equations to predict oral absorption of the training set and test set, respectively. Fit error of models for all chemical drugs (training set, $\mathrm{RMSEC}=7.76$; test set, RMSEC $=5.06$ ), acidic chemical drugs (training set RMSEC $=4.38$; test set RMSEC $=3.95$ ), basic chemical drugs (training set RMSEC $=3.08$; test set RMSEC $=2.98$ ) and natural chemical drugs (training set RMSEC $=3.00$ ) were relative low and descend gradually one by one, which suggested the better predictive ability was obtained and ionization of chemical drugs was considered necessarily. Fig. 1a-4a (supplementary files) and Fig. 1b-3b (supplementary files) show the activities of predicted value vs. experimental value of training set and test set for four models respectively. As can be observed, the better correlations were obtained and the correlation between predicted value and experimental value was improved one by one. Application developed regression methods for chemical drugs to predict oral absorption of bioactive ingredients of traditional Chinese medicines by biopartitioning micellar chromatography

The human intestinal absorption of drugs can be classified into three categories which are poor intestinal absorption ranged less than $30 \%$, high intestinal absorption ranged more than $80 \%$ and moderate intestinal absorption ranged between these two values $30 \%$ and $80 \%{ }^{28}$. The absorption in rats could be used as an alternative method to human absorption in pre-clinical oral absorption studies ${ }^{29}$. In this paper, animal intestinal absorption accounts to human oral absorption.

The ionization effect of bioactive ingredients of traditional Chinese medicines is common with the chemical drugs in membrane permeation when these acidic and basic bioactive ingredients dissociate under physiological $\mathrm{pH}$. On the basis of relationships between $\log \mathrm{k}$ and oral absorption for chemical drugs as mentioned above, oral absorption $\%$ of traditional Chinese medicines for bioactive ingredients were evaluated.

Prediction oral absorption of organic acid of traditional Chinese medicines for bioactive ingredients: Established regression models for all chemical and acidic chemical drugs were used to predict oral absorption \% of organic acids of traditional Chinese medicines for bioactive ingredients. Molecular descriptors and capacity factor $(\mathrm{k})$ for selected organic acid of bioactive ingredients were shown in Table-3 (supplementary files). The predicted oral absorption $\%$ calculated separately by eqns. 1 and 2, and experimental oral absorption $\%$ from literatures were listed in Table-4 (supplementary files). It was found that most of bioactive ingredients of traditional Chinese medicines in oral absorption predicted by model of acidic chemical drugs were better agreement with literature reported value than predicted by model of all chemical drugs.

Molecular descriptors selected for these structures are appropriate or not, which can affect prediction accuracy. Moreover, experimental condition to oral absorption date obtained may influence the accuracy in oral absorption. Therefore, not all of the predicted values of organic acids of traditional Chinese medicines for bioactive ingredients were consistent with literature value and acidic chemical drugs prediction model was not always better than model of all chemical drugs in predictability. Caffeic acid ${ }^{30}$ in the human body is passive diffusion through the deputy cells. Experiment founded that the oral absorption \% of caffeic acid was $95 \%$ obtained by orally administrated to healthy subjects. But its predicted oral absorption $\%$ by model of all chemical drugs was 79.56 and $70.09 \%$ predicted by model of acidic chemical drugs. Both of predicted values were lower than reported value. Besides, bioactive ingredients of traditional Chinese medicines in oral absorption $\%$ dates have not been studied clearly, so some of them can not get exact oral absorption \% value in this paper. For example, cinnamic acid, through models of acidic chemical drugs and all chemical drugs, the predictive value of cinnamic acid was 67.66 and $45.17 \%$, respectively. This can promote further study pharmacokinetics property of cinnamic acids and its analogues.

TABLE-3

DESCRIPTORS FOR ORGANIC ACIDIC OF TRADITIONAL CHINESE MEDICINES FOR BIOACTIVE INGREDIENTS

\begin{tabular}{|c|c|c|c|c|c|c|c|c|c|c|}
\hline No. & Compound & $1 / \mathrm{k}_{\mathrm{BMC}}$ & NHDL & Kappa_2 & NTR & $\mathrm{K} 3 \mathrm{~A}$ & JY & NAR & NSB & $\mathrm{NH}$ \\
\hline 1 & Chlorogenic acid & 0.39 & 6.00 & 8.79 & 1.00 & 4.56 & 2.09 & 1.00 & 1.00 & 18.00 \\
\hline 2 & Ferulaic acid & 0.21 & 2.00 & 5.78 & 1.00 & 6.07 & 2.98 & 1.00 & 1.00 & 8.00 \\
\hline 3 & Caffeic acid & 0.19 & 3.00 & 5.02 & 1.00 & 2.69 & 2.24 & 1.00 & 1.00 & 8.00 \\
\hline 4 & Cinnamic acid & 0.16 & 5.00 & 6.79 & 1.00 & 3.78 & 1.74 & 1.00 & 1.00 & 8.00 \\
\hline 5 & Rhein & 0.07 & 3.00 & 5.89 & 1.00 & 5.82 & 2.38 & 2.00 & 0.00 & 8.00 \\
\hline 6 & Salvianolic acid B & 0.24 & 9.00 & 19.43 & 2.00 & 8.83 & 1.49 & 2.00 & 1.00 & 30.00 \\
\hline 7 & Tanshinol & 0.39 & 7.00 & 12.19 & 2.00 & 6.88 & 2.81 & 1.00 & 0.00 & 9.00 \\
\hline
\end{tabular}




\begin{tabular}{|c|c|c|c|c|c|c|c|c|c|}
\hline \multicolumn{10}{|c|}{$\begin{array}{l}\text { TABLE-4 } \\
\text { EXPERIMENTAL ORAL ABSORPTION \% AND PREDICTED OA \% OF ORGANIC ACIDIC OF } \\
\text { TRADITIONAL CHINESE MEDICINES FOR BIOACTIVE INGREDIENTS }\end{array}$} \\
\hline \multirow[b]{2}{*}{ No. } & \multirow{2}{*}{\multicolumn{2}{|c|}{ Compound }} & \multicolumn{5}{|c|}{ Predicted (oral absorption \%) } & \multirow{2}{*}{\multicolumn{2}{|c|}{$\begin{array}{c}\text { Experimental } \\
\text { (oral absorption \%) }\end{array}$}} \\
\hline & & & \multicolumn{2}{|c|}{$\begin{array}{l}\text { By predictive model of } \\
\text { all chemical drugs }\end{array}$} & \multicolumn{2}{|c|}{$\begin{array}{l}\text { By predictive model of } \\
\text { acidic chemical drugs }\end{array}$} & & & \\
\hline 1 & Caffeotannic acid & & \multicolumn{2}{|c|}{55.87} & \multicolumn{2}{|r|}{43.87} & & \multicolumn{2}{|c|}{$33.33^{\mathrm{a}}$} \\
\hline 2 & Ferulaic acid & & \multicolumn{2}{|c|}{82.46} & \multicolumn{2}{|r|}{53.72} & & \multicolumn{2}{|c|}{$56.1 \pm 2.3^{b}$} \\
\hline 3 & Caffeic acid & & \multicolumn{2}{|c|}{79.56} & \multicolumn{2}{|r|}{70.09} & & \multicolumn{2}{|c|}{$95.00^{\mathrm{a}}$} \\
\hline 4 & Cinnamic acid & & \multicolumn{2}{|c|}{67.66} & \multicolumn{2}{|r|}{45.17} & & \multicolumn{2}{|c|}{-} \\
\hline 5 & Rhein & & \multicolumn{2}{|c|}{79.88} & \multicolumn{2}{|r|}{53.24} & & \multicolumn{2}{|c|}{$50-60^{c}$} \\
\hline 6 & Salvianolic acid B & & \multicolumn{2}{|c|}{16.06} & \multicolumn{2}{|r|}{10.82} & & \multicolumn{2}{|c|}{$4.46-14.55^{\mathrm{d}}$} \\
\hline 7 & Tanshinol & & \multicolumn{2}{|c|}{36.22} & \multicolumn{2}{|r|}{25.02} & & \multicolumn{2}{|c|}{$7.74-17.75^{\mathrm{d}}$} \\
\hline \multicolumn{10}{|c|}{ 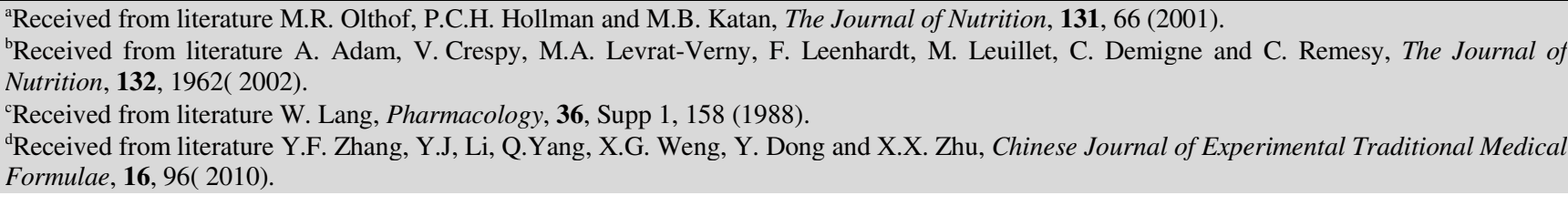 } \\
\hline \multicolumn{10}{|c|}{$\begin{array}{l}\text { TABLE-5 } \\
\text { NAL CHINE }\end{array}$} \\
\hline No. & Compound & $1 / \mathrm{k}_{\mathrm{BMC}}$ & NHDL & Kappa_2 & NTR & IC & MS & $\mathrm{NR}$ & PSA \\
\hline 1 & Sinomenine & 0.65 & 2.00 & 10.31 & 2.00 & 6.25 & -6.72 & 0.00 & 109.00 \\
\hline 2 & Berberine & 0.42 & 2.00 & 13.87 & 2.00 & 5.46 & -5.15 & 1.00 & 170.79 \\
\hline 3 & Aconitine & 0.08 & 2.00 & 7.39 & 0.00 & 4.44 & -3.82 & 2.00 & 109.51 \\
\hline 4 & Hypaconitine & 0.10 & 2.00 & 11.28 & 0.00 & 3.51 & -3.73 & 2.00 & 133.21 \\
\hline 5 & Colchicine & 0.79 & 1.00 & 10.54 & 1.00 & 3.93 & -4.70 & 0.00 & 83.09 \\
\hline 6 & Theophylline & 0.24 & 1.00 & 3.29 & 0.00 & 6.09 & 0.03 & 1.00 & 69.29 \\
\hline 7 & Tetrahydropalmatine & 0.50 & 1.00 & 9.16 & 2.00 & 4.93 & -5.49 & 0.00 & 70.16 \\
\hline
\end{tabular}

TABLE-6

EXPERIMENTAL ORAL ABSORPTION \% AND PREDICTED ORAL ABSORPTION \% OF ALKALOIDS OF TRADITIONAL CHINESE MEDICINES FOR BIOACTIVE INGREDIENTS

\begin{tabular}{|c|c|c|c|c|}
\hline \multirow[b]{2}{*}{ No. } & \multirow[b]{2}{*}{ Compound } & \multicolumn{2}{|c|}{ Predicted (oral absorption \%) } & \multirow{2}{*}{$\begin{array}{c}\text { Experimental } \\
\text { (oral absorption \%) }\end{array}$} \\
\hline & & $\begin{array}{l}\text { By predictive model of all } \\
\text { chemical drugs }\end{array}$ & $\begin{array}{l}\text { By predictive model of basic } \\
\text { chemical drugs }\end{array}$ & \\
\hline 1 & Sinomenine & 58.41 & 25.82 & Oral absorption $\% \leq 30^{\mathrm{a}}$ \\
\hline 2 & Berberine & 55.41 & 38.12 & Oral absorption $\% \leq 30^{\mathrm{b}}$ \\
\hline 3 & Aconitine & 90.17 & 85.58 & - \\
\hline 4 & Hypaconitine & 82.56 & 91.31 & - \\
\hline 5 & Colchicine & 69.18 & 59.63 & Oral bioavailability, $47 \pm 13^{c}$ \\
\hline 6 & Theophylline & 99.78 & 73.90 & $83 \pm 4^{\mathrm{d}}$ \\
\hline 7 & Tetrahydropalmatine & 67.52 & 45.43 & $40-50^{d}$ \\
\hline
\end{tabular}

${ }^{a}$ Received from literature W.Y. Chen, Y.D. Zhou, J.P. Kang, Q.B. Li and X.K. Fen, Study on absolute bioavailability of enteric-coated tablets of sinomenine. Chengyu Pharmaceutical Annual Conference Proceedings (2008) (in Chinese).

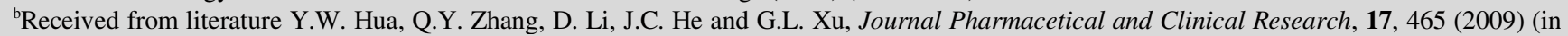
Chinese).

'Received from literature G.M. Ferron, M. Rochdi, W.J. Jusko and J.M. Scherrmann, Journal of Clinical Pharmacology, 36, 874 (1996).

${ }^{\mathrm{d}}$ Received from literature Z.Y. Hong, G.R. Fan, Y.F. Chai, X.P. Yin and T.Yu. Chinese Pharmaceutical Journal, 42, 1104 (2007) (in Chinese).

Prediction oral absorption of alkaloids of traditional Chinese medicines for bioactive ingredients: Models created by all chemical drugs and basic chemical drugs were used to predict oral absorption \% of alkaloids of traditional Chinese medicines for bioactive ingredients. The molecular descriptors and value of capacity factor $(\mathrm{k})$ for selected alkaloids are showed in Table-5 (supplementary files). The predicted oral absorption $\%$ calculated separately by eqns. 1 and 3 , and experimental oral absorption \% from literatures are showed in Table-6.

Due to poor oral absorption and inappropriate half-life time, most of bioactive ingredients of traditional Chinese medicines need to be orally administrated many times and choose to be administrated through transdermal, intramuscular and intravenous. Adding to not clear research on the pharmacokinetics particular absorption process, most of alkaloids of traditional Chinese medicines for bioactive ingredients can not obtain available oral absorption \%. For example, sinomenine and berberine have poor oral absorption. Tetrahydropalmatine absorbed in the rat small intestine was probable mainly passive diffusion. However, their oral absorption \% predicted by model of basic chemical drug was better consistent with literature reported than model of all chemical drugs (Table-6 in supplementary files). Oral bioavailability is particularly important for lead optimization considering the importance of developing 


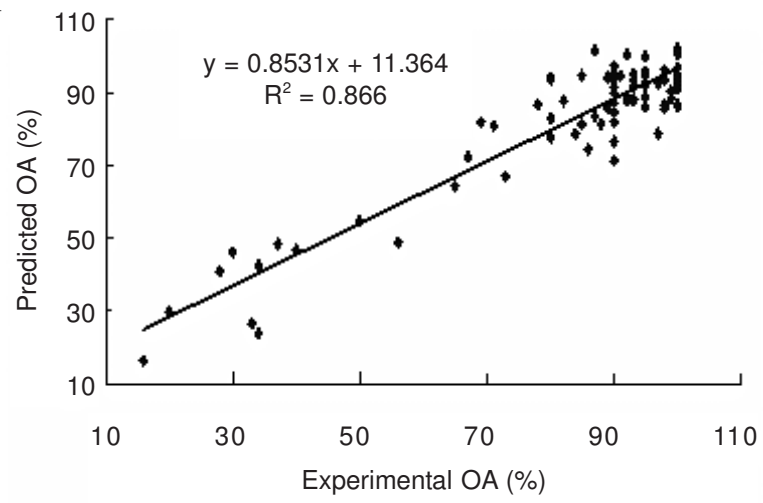

a. Training set

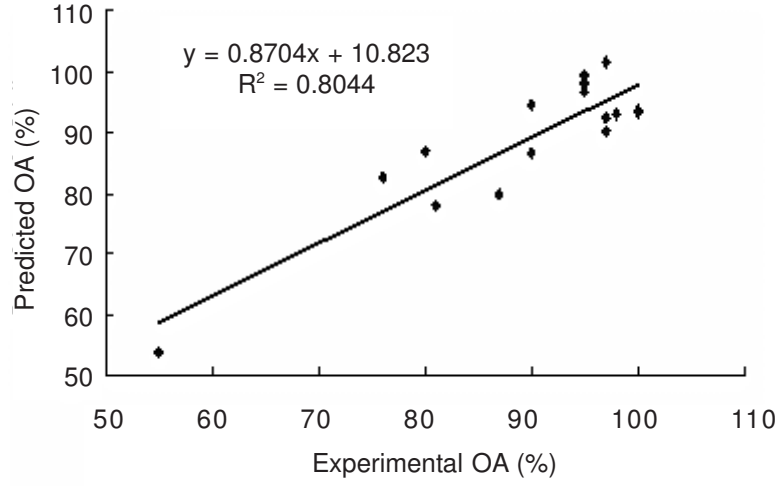

b. Test set

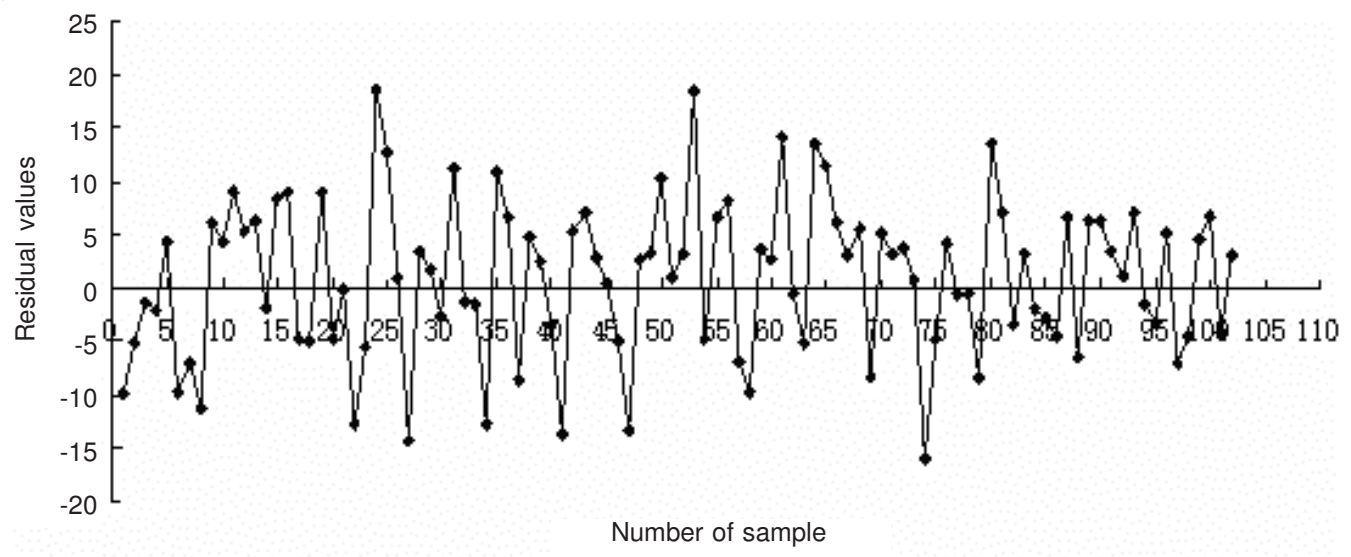

c. Plot of residual values

Fig. 1. (a) and (b) show that predicted value calculated by eqn. $1 \mathrm{vs}$. experimental value of training set and test set of all chemical drugs, respectively. $\mathrm{c}$ represents the residual values [Experimental oral absorption (\%)-Predicted oral absorption (\%)] of all chemical drugs, $\mathrm{a}, \mathrm{b}$ and $\mathrm{c}$ are to validate the predicted ability of model constructed

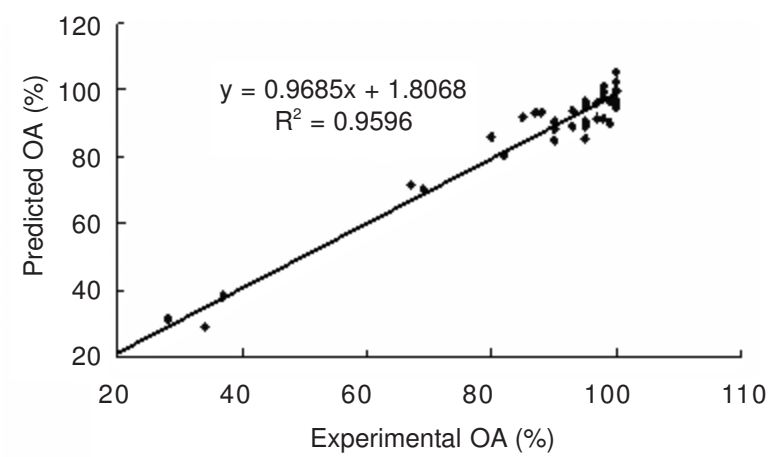

a. Training set

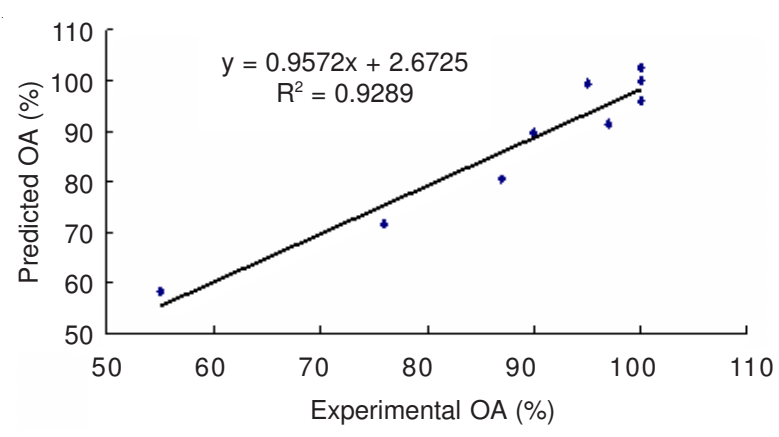

b. Test set

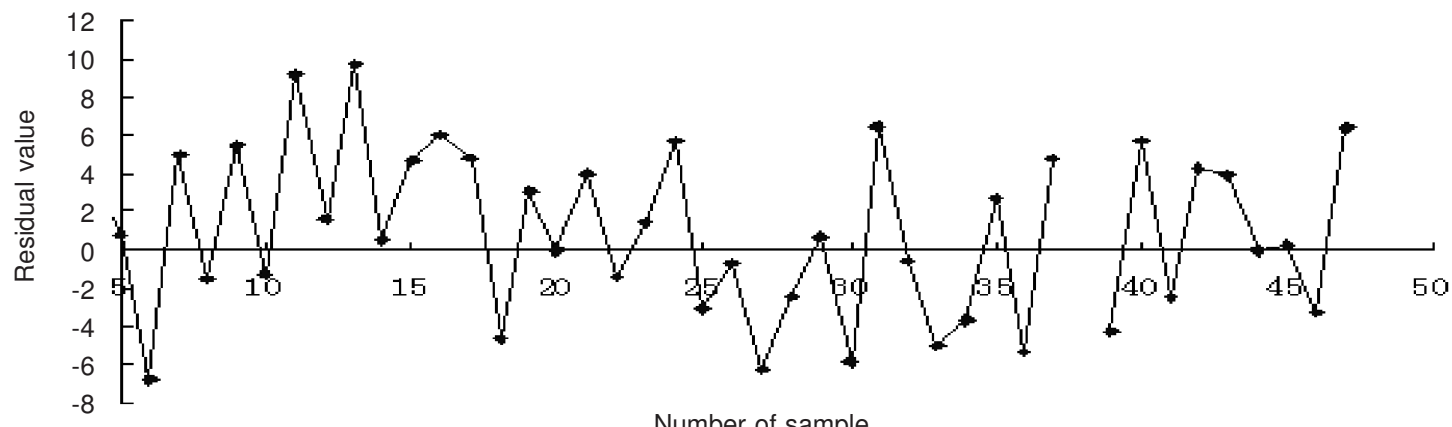

Number of sample

c. Plot of residual values

Fig. 2. (a) and (b) show that predicted value calculated by eqn. 2 vs. experimental value of training set and test set of acidic chemical drugs, respectively. $\mathrm{c}$ represents the residual values [Experimental oral absorption (\%)-Predicted oral absorption (\%)] of acidic chemical drugs, a, b and c are to validate the predicted ability of model constructed 


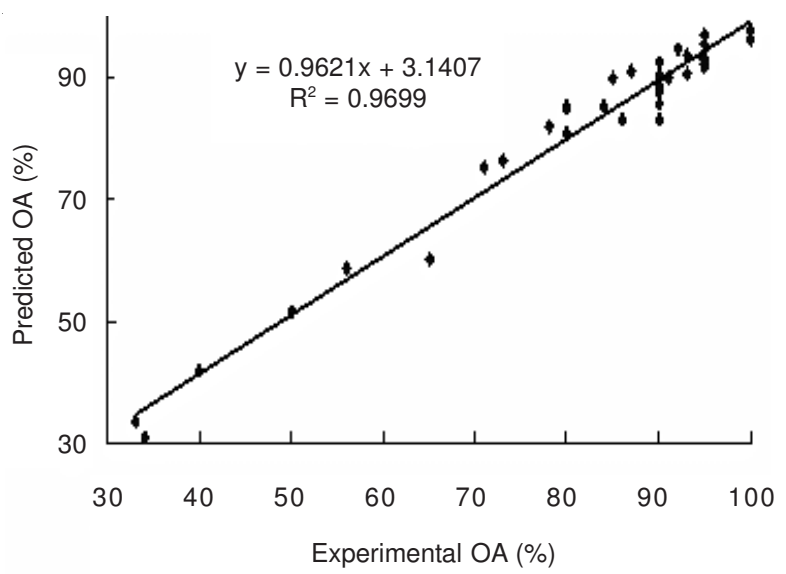

a. Training set

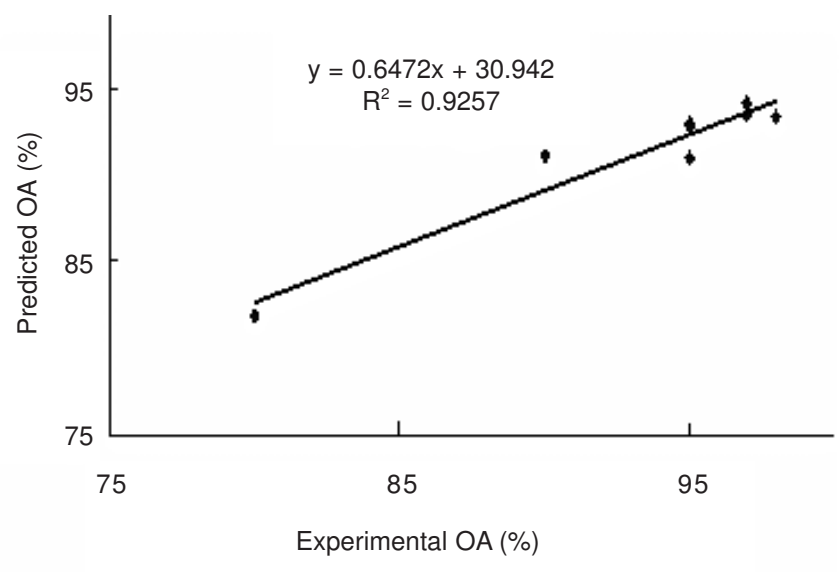

b. Test set

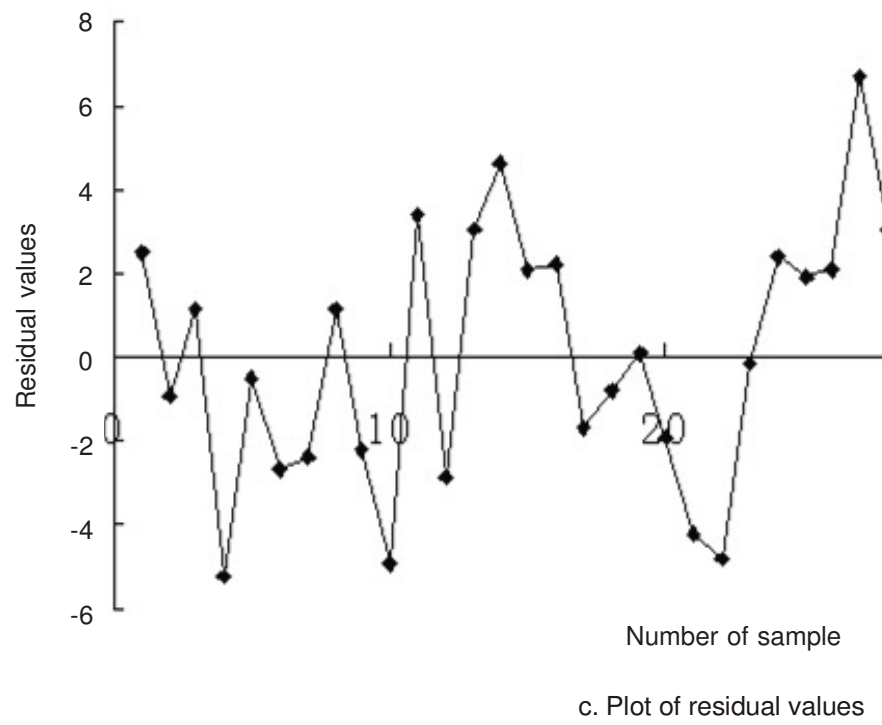

Fig. 3. (a) and (b) show that predicted value calculated by eqn. 3 vs. experimental value of training set and test set of basic chemical drugs, respectively. c represents the residual values [Experimental oral absorption (\%)-Predicted oral absorption (\%)] of basic chemical drugs, a, b and c are to validate the predicted ability of model constructed

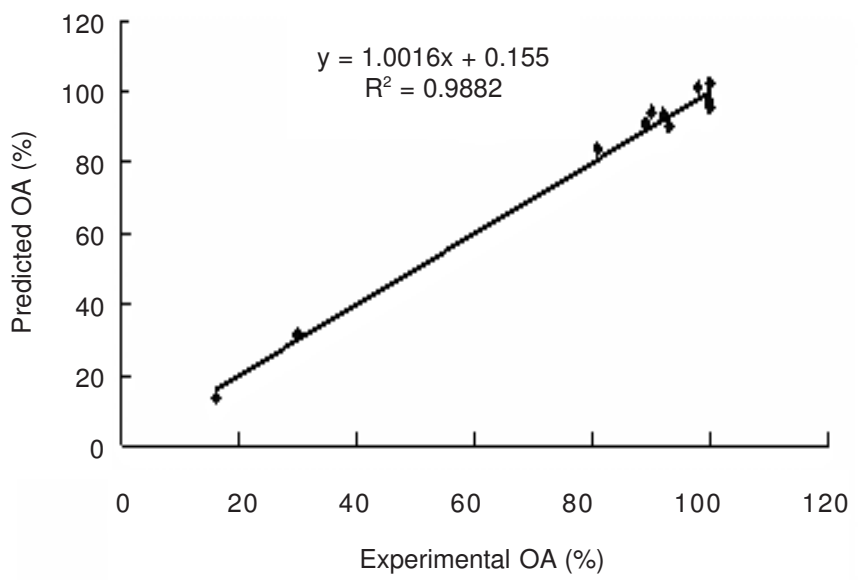

a. Training set

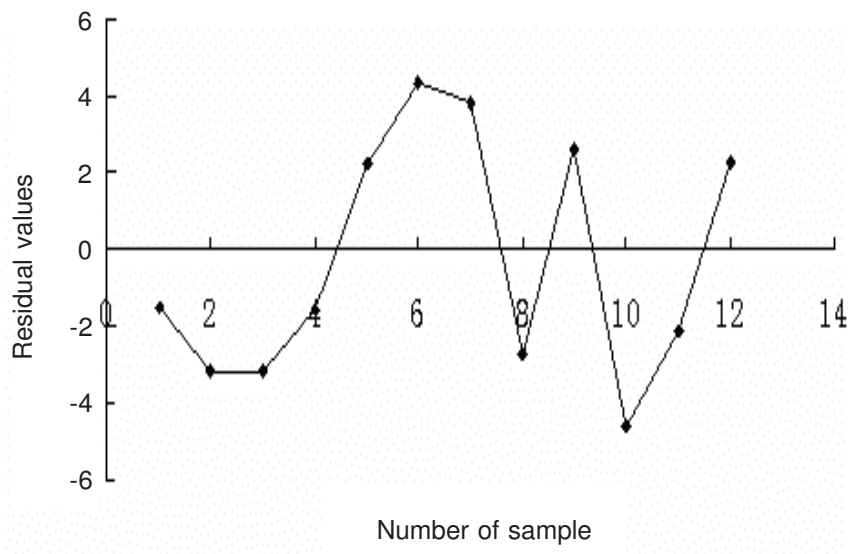

b. Test set

Fig. 4. (a) shows that predicted value calculated by eqn. 4 vs. experimental value of training set natural chemical drugs, respectively. (b) represents the residual values [Experimental oral absorption (\%)-Predicted oral absorption (\%)] of natural chemical drugs, a and b are to validate the predicted ability of model constructed 
orally administered commercial products. After oral administration, the bioavailability was low, the absorption should be equal to or higher than the values of bioavailability because following absorption from the gastrointestinal tract, the drug passes directly to the liver via the hepatic portal vein where it may be extensively metabolized before reaching the systemic circulation $^{31}$, such as berberine and colchicines ${ }^{32}$. The literature just reported hydrochloride bioavailability of berberine was about $10 \%$. Colchicine belongs to the class of moderate oral absorption which ranges form 30 to $80 \%$. Its oral bioavailability was about $40-50 \%$. Their oral absorption $\%$ prediction values were higher than their oral bioavailability reported value (Table-6) and predicted value from model of basic chemical were more close to reported value than model of all chemical drugs. Similarly, prediction from basic chemical drugs model was more consistent with literature reported.

Aconite root is mainly composed of aconitine and hypaconitine that are diester diterpenoid alkaloids and the nature are unstable, decomposing in the water immediately. Previous studies $^{33}$ applied Caco- 2 cell model to simulate the human body's intestinal absorption process, predict the oral absorption of chemicals and use this model to carry out study of absorption for aconitine and hypaconitine. The research revealed that the apparent permeability coefficients $\left(\mathrm{P}_{\text {app }}\right)$ value of aconitine and hypaconitine have good oral absorption because their $\mathrm{P}_{\text {app }}$ values were more than $1 \times 10^{-6} \mathrm{~cm} / \mathrm{s}$. $\mathrm{P}_{\text {app }}$ which associates with the oral absorption $\%$ of chemical drugs directly reflects the drug absorption. There is complete absorption when Papp value of drug is large than $1 \times 10^{-6} \mathrm{~cm} / \mathrm{s}^{34}$. Predicted oral absorption $\%$ of aconitine by models of all chemical drugs and basic chemical drugs was 90.17 and $85.58 \%$ and hypaconitine was 82.56 and $91.31 \%$.

\section{Conclusion}

In this study, when the acid-base property of drugs was taken into consideration to predict oral absorption of diverse structural drugs by biopartitioning micellar chromatography technique, capacity factor $\left(\mathrm{k}_{\mathrm{BMC}}\right)$ measured on biopartitioning micellar chromatography column at $\mathrm{pH} 7.4$ and molecular structure descriptors were better correlated with oral absorption \% of drugs. At the same time, constructed models process certainly fit ability and statistics significance. The absorption model allows prediction of oral absorption can be used to determine how to modify drug structure to improve absorption. In summary, four prediction models of chemical drugs constructed are robust and reliable. So application those in prediction oral absorption of monomers of active ingredients in traditional Chinese medicines are practical.

We just preliminarily studied retention on the biopartitioning micellar chromatography and molecular descriptors of monomers of traditional Chinese medicines for bioactive ingredient through model of chemical drugs to predict oral absorption. However, absorption is a complex kinetic process that is dependent on numerous biochemical, physiological and physico-chemical factors. Experimental error for obtaining absorption value is quite high, especially for low absorption. It is unrealistic to attempt to obtain prediction bioactive values which agree well with reported values which were obtained from animals. So large data set and complete molecular descriptors and accurate literature value about bioactive should be considered when construct prediction model.

In following research, we aim to further study oral absorption. To mimic an environment, which more closely resembles the conditions encountered as the substance moves through the gastrointestinal tract and knowing the importance of $\mathrm{pH}$ conditions to retention of acidic compounds, capacity factor measurements will be made at varying $\mathrm{pH}$ values, from $\mathrm{pH}$ 4.5-7.4. And appropriate molecular descriptors of different aspects will be selected to construct model and evaluate predictive ability by RMSEC (the root-mean-square error of calibration) and RMSECV (root-mean-square error of crossvalidation). Besides, screening bioactive ingredients from traditional Chinese herbal medicine will apply high throughput screening method biopartitioning micellar chromatography by means of combinatorial chemistry which are widely used in drug research and discovery in conjunction with mathematical statistics method to construct QRAR/QSAR models to predict oral absorption, distribution, metabolism and elimination (ADME) of traditional Chinese medicine compound preparation.

\section{ACKNOWLEDGEMENTS}

The authors greatly acknowledge the highly professional technical support and statistical help from Prof. ShengYong Yang and Hui Zhang (State Key Laboratory of Biotherapy, Sichuan University) for valuable discussion.

\section{REFERENCES}

1. J. Kotech, S. Shah, I. Rathod and G. Subbaiah, Int. J. Pharm., 360, 96 (2008).

2. Y.C. Martin, J. Med. Chem., 24, 229 (1981)

3. J. Kotech, S. Shah, I. Rathod and G. Subbaiah, Int. J. Pharm., 333, 127 (2007).

4. R. Lu, J. Sun, Y.J. Wang and G. He, Chromatographia, 70, 21 (2009).

5. M.D. Wessel, P.C. Jurs, J.W. Tolan and S.M. Muskal, J. Chem. Inf. Model., 38, 726 (1998).

6. U. Norinder, T. Osterberg and P. Artursson, Pharm. Res., 14, 1786 (1997).

7. C.A. Lipinski, F. Lombardo, B.W. Dominy and P.J. Feeney, Adv. Drug Deliver. Rev., 23, 23 (1997).

8. K. Palm, K. Luthman, A. Ungell, G. Strandlund, F. Beigi, P. Lundahl and P. Artursson, J. Med. Chem., 41, 5382 (1998).

9. J. Kelder, P.D.J. Grootenhuis, D.M. Bayada, L.P.C. Delbressine and J. Ploemen, Pharm. Res., l16, 1514 (1999).

10. M.V. Shah, K.L. Audus and R.T. Borchardt, Pharm. Res., 6, 624 (1989).

11. M. Molero-Monfort, L. Escuder-Gilabert, R.M. Villanueva-Camañas, S. Sagrado and M.J. Medina-Hernández, J. Chromatogr. B, 753, 225 (2001).

12. L. Escuder-Gilabert, J.J. Martinez-Pla, S. Sagrado, R.M. Villanueva-Camañas and M.J. Medina-Hernández, J. Chromatogr. B, 797, 21 (2003).

13. L. Escuder-Gilabert, J.J. Martínez-Pla, S. Sagrado, R.M. VillanuevaCamañas and M.J. Medina-Hernández, Anal. Chim. Acta, 448, 173 (2001).

14. J.J. Martínez-Pla, Y. Martín-Biosca, S. Sagrado, R.M. VillanuevaCamañas and M.J. Medina-Hernández, J. Biomed. Chromatogr., 17, 530 (2003)

15. L. Escuder-Gilabert, J.J. Martínez-Pla, S. Sagrado, R.M. VillanuevaCamañas and M.J. Medina-Hernánde, J. Chromatogr. B, 807, 193 (2004).

16. R. Lu, J. Sun, Y.J. Wang, H.Y. Li, J.F. Liu, L. Fang and Z.G. He, J. Chromatogr. A, 1216, 5190 (2009).

17. S.R. Wang, Y. Chen, L.P. Wu, W.J. Miao, M.J. Xiong, C. Chen, Z.R. Zhong and L.M. Ye, J. Pharmaceut. Biomed., 2, 243 (2008).

18. L.P. Wu, Y. Chen, S.R. Wang, C. Chen and L.M. Ye, J. Pharmaceut. Biomed., 46, 243 (2008).

19. L.P. Wu, Y. Chen, C. Chen, S.R. Wang, C. Chen and L.M. Ye, Biomed. 
Chromatogr, 22, 106 (2008)

20. L.P. Wu, L.M. Ye, C. Chen, J.Q. Wu and Y. Chen, Biomed. Chromatogr., 22, 606 (2008).

21. L.P. Wu, Y. Cui, M.J. Xiong, S.R. Wang, C. Chen and L.M. Ye, Biomed. Chromatogr., 22, 1243 (2008).

22. S.R. Wang, C. Chen, M.J. Xiong, L.P. Wu and L.M. Ye, J. Chromatogr. Sci., 48, 134 (2010).

23. Z.X. Hu, W.N. Zhang, H.B. He, Y.Q. Feng and S.L. Da, Chromatographia, 69, 637 (2009).

24. M. Molero-Monfort, L. Escuder-Gilabert, R.M. Villanueva-Camañas, S. Sagrado and M.J. Medina-Hernández, J. Chromatogr. B: Biomed. Sci. Appl., 753, 225 (2001)

25. T. Österberg, M. Svensson and P. Lundahl, Eur. J. Pharm. Sci., 12, 427 (2001).

26. X.Y. Liu, C. Nakamura, Q. Yang, N. Kamo and J. Miyake, J. Chromatogr., 961, 113 (2002).
27. F. Barbato, M.I. LaRotonda and F. Quaglia, Pharm. Res., 14, 1699 (1997).

28. M.A. Cabrera Pérez, M.B. Sanz, L.R. Torres, R.G. Ávalos, M. González and H. González Díaz, Eur. J. Med. Chem., 39, 905 (2004).

29. Y.H. Zhao, M.H. Abrahama, J. Le, A. Hersey, C.N. Luscombe, G. Beck, B. Sherborne and I. Cooper, J. Pharm. Sci., 90, 749 ( 2001).

30. M.R. Olthof, P.C.H. Hollman and M.B. Katan, J. Nutr., 131, 66 (2001).

31. Y.H. Zhao, J. Le, M.H. Abraham, A. Hersey, P.J. Eddershaw, C.N. Luscombe, D. Boutina, G. Beck, B. Sherborne, I. Cooper and J.A. Platts, J. Pharm. Sci., 90, 749 (2001).

32. G.M. Ferron, M. Rochdi, W.J. Jusko and J.M. Scherrmann, J. Clin. Pharmacol., 36, 874 (1996)

33. N. Li, M.D. Thesis, The In Vitro Study on Absorption and Mechanism of Diester-type Alkaloids in Aconite Extract, Ji Lin University (2009).

34. M.C. Grès, B. Julian, M. Bourrié, V. Meunier, C. Roques, M. Berger, X. Boulenc, Y. Berger and F. Gérard, Pharm. Res., 15, 726 (1998). 